Proc. 15th Int. Conference on Defects Recognition, Imaging and Physics in Semiconductors, Warsaw, Poland 2013

\title{
Raman Study of Multicrystalline Silicon Wafers Produced by the RST Process
}

\author{
A. Tejero ${ }^{a}$, E. Tupin ${ }^{b}$, M.A. GonzÁlez ${ }^{a, *}$, O. Martínez $^{a}$, J. Jiménez ${ }^{a}$, C. Belouet $^{b}$ \\ AND C. BAILlis ${ }^{c}$ \\ ${ }^{a}$ GdS-Optronlab, Dpto. Física Materia Condensada, Parque Científico Univ. de Valladolid, 47011 Valladolid, Spain \\ ${ }^{b}$ Solarforce, 1 rue du Dauphin, 38300 Bourgoin-Jallieu, France \\ ${ }^{c}$ EC2-Modélisation, 66 bd Niels Bohr CS 52132, 69603 Villeurbanne, France
}

\begin{abstract}
In the silicon ribbon on a sacrificial template process silicon is deposited on both sides of a carbon ribbon, thus forming a Si/carbon/Si trilayer. The fast cooling of the ribbon in large temperature gradients generates stresses that are detrimental to both the electrical performance and the mechanical behaviour of the wafers. The assessment of the stresses is crucial for the setting-up of thermal treatments allowing for the stress relaxation of the wafers, prior to the cell fabrication. We present an analysis of the stress in the as-grown trilayer by a simulation of the thermomechanical behaviour of the cooling ribbon. Experimental measurements of the stress in as-grown and annealed trilayers are also presented. The results permit to establish the conditions for optimized growth and annealing.
\end{abstract}

DOI: $10.12693 /$ APhysPolA.125.1006

PACS: 78.30.Am, 61.72.Ff, 88.40.jj

\section{Introduction}

Silicon ribbon technology has emerged as a cost-effective method alternative to other multicrystalline $\mathrm{Si}$ growth processes [1, 2]. Among these technologies, the silicon ribbon on a sacrificial carbon template (RST) technique developed by Solarforce has emerged as the ribbon technology able to produce quasi flat thin wafers down to $50 \mu \mathrm{m}$ at high pull rates $(\geq 5 \mathrm{~cm} / \mathrm{ms})$; a conversion efficiency of $16 \%$ has recently been obtained on RST wafers $80 \mu \mathrm{m}$ thick $[3,4]$. The vertical growth configuration at high pulling speeds of this technique results in large temperature gradients, in excess of $1000{ }^{\circ} \mathrm{C} / \mathrm{cm}$ at the solid liquid interface; such large gradients build up thermal stresses, which eventually have detrimental consequences on both the electrical properties of the ribbon wafers, and the mechanical yield of the process. Two challenges for further improvement of the RST technology are the optimization of the electrical performances to reach conversion efficiencies above $17 \%$, and the reduction of the residual stresses.

We present herein an analysis of the residual stress in the RST-Si by using two approaches; namely: (i) a simulation of the thermomechanical behaviour of the cooling ribbon using the Abaqus software, which takes into account the ribbon dimensions and the experimental pulling conditions and (ii) the measurement of the residual stresses on the free silicon ribbon surface by $\mu$-Raman spectroscopy. The thermomechanical simulation took into account the viscoelastic properties of silicon and gave

*corresponding author; e-mail: oscar@fmc.uva.es the maximum deformation of the cooled ribbon in the absence of post-annealing treatments. $\mu$-Raman scans and maps were made on different samples, as-grown (i.e. prior to the removal of the carbon ribbon) and after post growth annealing treatments, either off-line (or ex situ) and in-line with growth.

\section{Experimental and samples}

In the RST process, a carbon ribbon, composed of a soft low density graphite ribbon coated on both sides by a high density pyrocarbon layer deposited by chemical vapor deposition (CVD) is vertically pulled upwards throughout a crucible filled with molten silicon. The freezing meniscus thus formed on both sides of the carbon ribbon yields a $\mathrm{Si} /$ carbon/Si trilayer coming out of the growth chamber. Afterwards, the trilayer is laser cut and exposed to an oxygen-rich gas at high temperature $\left(\approx 1000^{\circ} \mathrm{C}\right)$ in order to eliminate (burn-off step) the carbon ribbon. Two thin $\mathrm{Si}$ wafers $(\leq 100 \mu \mathrm{m})$ are obtained from each trilayer after the burn-off step. Then, the self-standing Si wafers are wet or plasma etched prior to starting the fabrication of the solar cells.

The crystalline texture of the silicon film consists of large grains elongated along the pulling direction and continuous down to the carbon substrate; the boundaries between these grains (GBs) can be observed in the optical microscope together with twin structures aligned along the pulling axis [5], Fig. 1.

The stresses, the warpage of the RST ribbon and the silicon thickness across the ribbon were estimated by means of thermomechanical simulations using the Abaqus software. An iterative method described in a previous work was used to take into account the occurrence of asymmetrical growth [6]. Due to the lack of data on the 


\section{SURFACE}

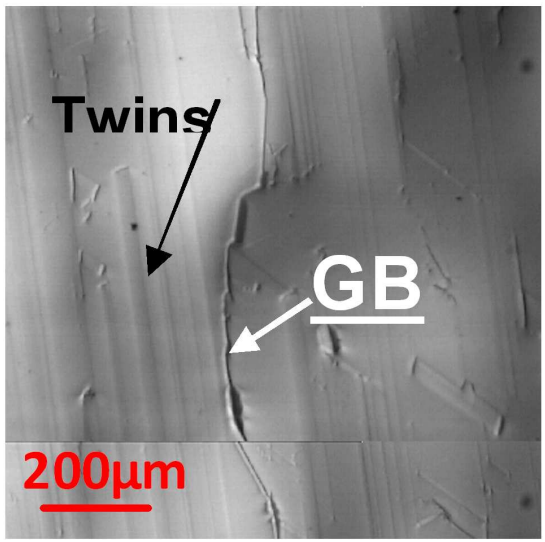

Fig. 1. Optical image of an as-grown sample showing a grain boundary (GB) along the pulling axis, and twins.

viscoelastic properties of silicon at high temperature, the simulation used the experimental data obtained by Graham et al. on silicon compressed at temperatures ranging from 1100 to $1380^{\circ} \mathrm{C}$ at several strain rates [7]. A further simplification was the use of a vertical symmetry plane normal to the ribbon glide plane. The relaxation effect, presently under investigation, was not taken into account in the simulations presented here. The measure of the ribbon warpage presented in this paper is the maximal deformation, called $\Delta z$, in the direction of the ribbon normal, we label this deformation as deflection. Reference calculations were made for thicknesses of the carbon ribbon, pyrocarbon coating and silicon thicknesses of 270 , 4 , and $100 \mu \mathrm{m}$, respectively.

The experimental assessment of the stress in the silicon films was carried out with a Labram UVHR 800 Raman micro-spectrometer from Horiba-Jobin Yvon. The excitation was done with a helium neon laser $(632 \mathrm{~nm})$ for which the penetration depth $(\approx 3 \mu \mathrm{m})$ was deep enough to minimize the influence of the surface. The smooth surface of the RST samples permitted to perform the measurements without additional surface preparation. The use of a careful spectral reference setup was mandatory because of the small Raman peak shift; a neon lamp located inside the optical entrance of the spectrometer, just ahead of the entrance slit, warranted a very stable spectral reference insensitive to the beam alignment. A detailed calculation of the stress from the Raman shift would require the knowledge of the crystal orientation [8]; the stress was approximated using the Raman shift induced by biaxial stress in the (100) plane $(\sigma=250$ $\left.\left(\mathrm{MPa} / \mathrm{cm}^{-1}\right) * \Delta \omega\right)[9]$ from which the residual stresses were calculated. Raman maps were obtained in different zones of the samples both as-grown and after annealing; the same zones of the as-grown and annealed samples were mapped by $\mu$-Raman spectroscopy in order to evaluate the influence of the annealing step on the residual stress relaxation.

\section{Results and discussion}

The simulation of the stress in RST Si predicted a rapid increase of the amplitude of the deformation $\Delta z$ beyond acceptable limits $(>1 \mathrm{~mm}$ ) for ribbon widths above $55 \mathrm{~mm}$ in good agreement with the experimental trend, even though experimental results showed a less critical dependence, Fig. 2. The simulation also predicted an optimum scenario with $\Delta z=0$ for $55 \mathrm{~mm}$ ribbon width and Si films $100 \mu \mathrm{m}$ thick, which was experimentally approached up to a width of $65 \mathrm{~mm}$. A very interesting output of the simulation work was the reduction of $\Delta z$ as the pull rate increased, giving silicon thicknesses down to $50 \mu \mathrm{m}$, a prediction in agreement with experimental findings.

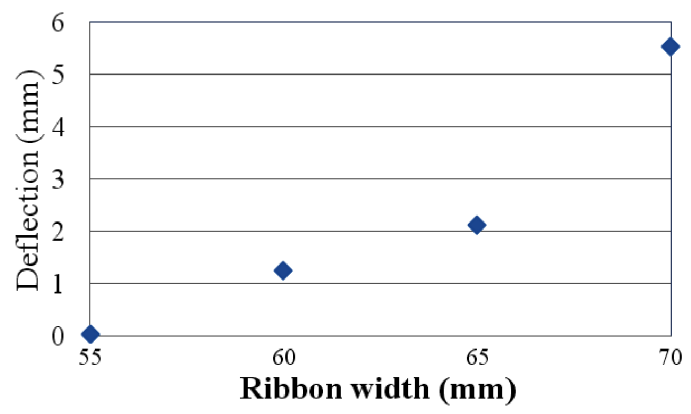

Fig. 2. Dependence of the deformation $(\Delta z)$, labeled deflection, on the ribbon width.

The macroscopic average tensile stress calculated in the absence of relaxation was around $30 \mathrm{MPa}$, in good agreement with the stress estimated from the Raman shift in as-grown samples.

The Raman study was done in two steps; firstly, the Raman spectra were recorded on as-grown and subsequently, in the same regions of the sample after ex situ annealing. The results of the Raman spectra showed a remarkable relaxation effect for annealing temperature $T_{\mathrm{p}}$ and duration $\tau_{\mathrm{p}}$ values of $c a .900{ }^{\circ} \mathrm{C}$ and $10 \mathrm{~min}$., respectively. The local stresses were measured by scanning the laser beam over well identified crystal defects, e.g. GBs; an example is shown in Fig. 3 which presents stress distribution maps obtained from the Raman shift before and after the ex situ annealing on an area of the sample with a GB. One observes tensile stress on one side of the grain boundary and compressive stress on the other side, superimposed to the overall tensile stress of the as-grown trilayer.

The trilayer sample annealed ex situ presented a relaxation of the macroscopic tensile stress, while the local stresses on either side of the grain boundary presented a similar distribution to that measured prior to the annealing, but smoothed out with respect to the as-grown stress distribution, see Fig. 3. The stress relaxation is summarized in the histograms extracted from the two stress maps of Fig. 3, as-grown and annealed samples respectively, Fig. $3 \mathrm{~b}$ and $\mathrm{c}$. 


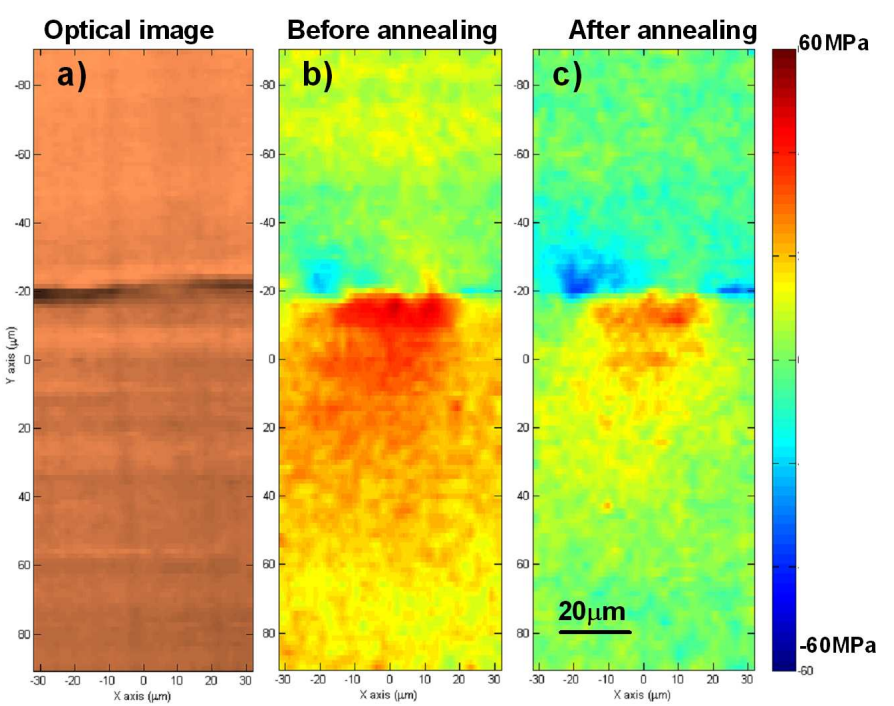

Fig. 3. (a) Optical image showing a GB, (b) stress map measured from Raman shift in the same trilayer sample as-grown, and (c) stress map after ex situ annealing.

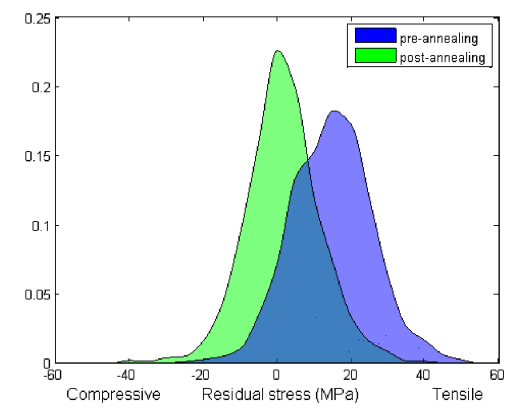

Fig. 4. Histograms representing the stress distribution before and after ex situ annealing, extracted from the data of Fig. 3, showing stress relaxation by annealing (see text).

The histograms of Fig. 4 show that after thermal treatment the tensile stress was relaxed and the stress distribution narrowed, accounting for the smoothing of the stress distribution around the grain boundary. It was also found that the fragile behaviour of the ribbon during laser cutting could be considerably reduced by the ex situ annealing step. These experimental results were used to optimise the in-line annealing of the RST ribbon.

A post-heater was mounted on the ribbon puller in order to relax the strains induced in the silicon films by the rapid cooling of the ribbon. The position $H_{\mathrm{p}}$ of the post heater above the freezing front and the in-line annealing parameters (plateau temperature in the post-heater $T_{\mathrm{p}}$ and duration $\tau_{\mathrm{p}}$ ) are the parameters which control the relaxation step and define the post heater configuration ( $T_{\mathrm{p}}$ is actually the closest value to constant temperature inside the post heater).

The RST ribbons undergoing an in-line annealing (relaxation) were evaluated by two means, namely: the measurement of the strain field by $\mu$-Raman spectroscopy, performed directly on the ribbon coming out of the puller and (ii) the measurement of the laser cutting yield, which represents the fraction of crack-free wafers, $50 \mathrm{~mm}$ wide, potentially extracted from ribbons $55 \mathrm{~mm}$ in width. Several configurations $\left(H_{\mathrm{p}}, T_{\mathrm{p}}\right.$ and $\left.\tau_{\mathrm{p}}\right)$ of the post heater have been tested. Examples of temperature profiles are shown in Fig. 5.

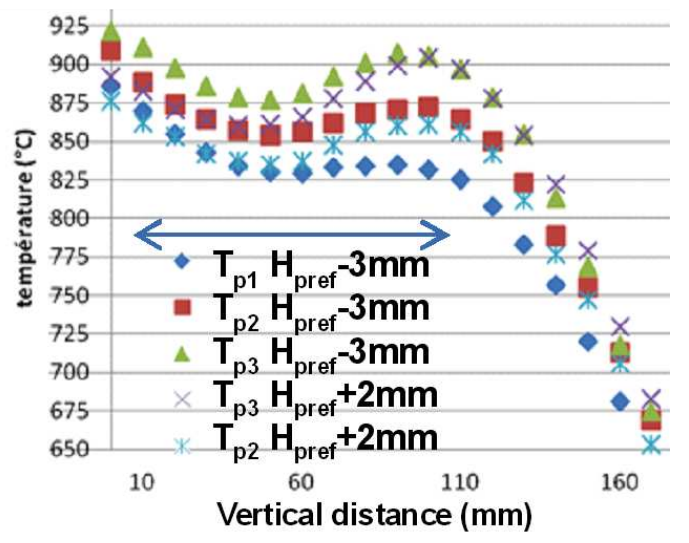

Fig. 5. Temperature profiles of the post heater for three different plateau temperatures $\left(T_{\mathrm{p} 1}, T_{\mathrm{p} 2}\right.$ and $\left.T_{\mathrm{p} 3}\right)$ and different positions with respect to the optimal position, labeled reference position, $H_{\text {pref }}$; the double arrow gives the approximate position of the active part of the post heater.

A remarkable increase of the laser cutting yield after in-line annealing was observed. The yield was increased from $50 \%$, without annealing, to values close to $100 \%$ under the best annealing conditions.

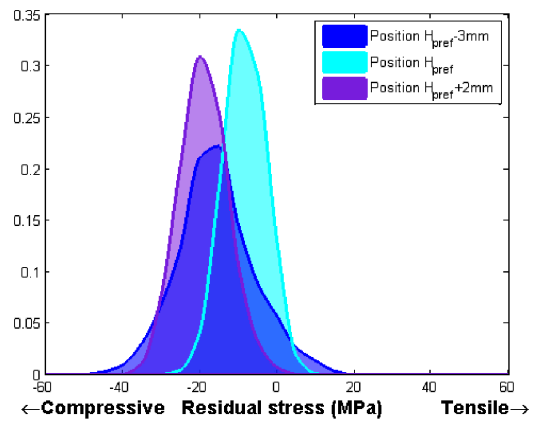

Fig. 6. Histograms representing the stress distribution obtained from the Raman shift after in-line annealing for three different post-heater positions. For the reference $H_{\mathrm{p}}$ (optimal position) the stress is relaxed; when one modifies the position, compressive stress is induced.

On the other hand, the Raman results showed that the in-line annealing relaxed the stress, which surprisingly became slightly compressive. A significant relaxation of the average stress was observed for increasing values of $T_{\mathrm{p}}$, and the adequate choice of the post heater position, $H_{\mathrm{p}}$, Fig. 6. For example, with the annealing 
configuration corresponding to a profile with parameters ( $H_{\text {pref }}, T_{\mathrm{p}} \approx 850{ }^{\circ} \mathrm{C}$ and $\tau_{\mathrm{p}}=4 \mathrm{~min}$ ), a laser cutting yield ca. $97 \%$ was achieved.

The influence of the position of the post-heater is summarized in Fig. 6, where the stress distribution histograms are represented for three different post heater positions. For the optimal position, $H_{\text {pref }}$, the stress is relaxed, while for positions above and below compression is observed. This behaviour depends as well on $T_{\mathrm{p}}$ and $\tau_{\mathrm{p}}$; in particular, high $T_{\mathrm{p}}$ presents compressive trends, while low $T_{\mathrm{p}}$ shows tensile trends; therefore, the combination of the three parameters should permit a significant relaxation of the built in residual stresses.

\section{Conclusion}

The combination of thermomechanical simulation of the cooling ribbon and of the experimental assessment of the stresses in RST Si by $\mu$-Raman spectroscopy has been demonstrated to constitute a powerful tool to improve the technology by providing better control of the growth parameters and of the optimization of the post growth annealing step; further research following this work will include the simulation of the relaxation step and the optimization of the temperature, $T_{\mathrm{p}}$, and the time, $\tau_{\mathrm{p}}$ in the post-heater setup.

\section{Acknowledgments}

The authors from Solarforce wish to thank the Agence De l'Environnement et de la Maîtrise de l'Energie ADEME (for supporting this work and E. Benedetti,
S. Lebsir and Y. Delaup for the experimental part of the work. The Spanish group was partially funded by the Research Projects VA166A11-2 "Consejería de Educación, Junta de Castilla y León", Spain) and IPT-420000-2010-022 (INNPACTO program, "Ministerio de Ciencia e Innovación", Spain)

\section{References}

[1] C. Belouet, J. Cryst. Growth 82, 110 (1987).

[2] G. Sarau, S. Christiansen, M. Holla, W. Seifert, Solar Energy Mater. Solar Cells 95, 2264 (2011).

[3] P. Keller, F. De Moro, S. Seren, G. Hahn, Energy Procedia 38, 576 (2013).

[4] F. De Moro, A. Focsa, K. Derbouz, A. Slaoui, N. Auriac, H. Lignier, P. Keller, Phys. Status Solidi C 9, 2092 (2012).

[5] A. Gervais, R. Sharko, T. Moudda-Azzem, C. Belouet, J. Cryst. Growth 82, 209 (1987).

[6] C. Bigot, C. Baillis, E. Jolivet, M. Coret, R. Varrot, C. Belouet in: Proc. 6th Int. Workshop on Crystalline Silicon Solar Cells, Aix-les-Bains (France), 2012.

[7] C.D. Graham, D.P. Pope, S. Kulkarni, Wolf, NASA report: Hot forming of silicon sheet, silicon sheet growth development for large area silicon sheet task of the low cost silicon solar array project, ERDA/JPL954506-78/1 DC UC-6.

[8] M. Becker, H. Scheel, S. Christiansen, H.P. Strunk, J. Appl. Phys. 101, 063531 (2007).

[9] I. DeWolf, Semicond. Sci. Technol. 11, 139 (1996). 